\title{
Novel Ion Trap Scan Modes to Develop Criteria for On-Site Detection of Sulfonamide Antibiotics
}

\author{
Lucas J. Szalwinski, Yanyang Hu, Nicolás M. Morato, R. Graham Cooks, * and Gert IJ. Salentijn*
}

Cite This: Anal. Chem. 2021, 93, 13904-13911

Read Online

ABSTRACT: Advances in ambient ionization techniques have facilitated the direct analysis of complex mixtures without sample preparation. Significant attention has been given to innovating ionization methods so that multiple options are now available, allowing for ready selection of the best methods for particular analyte classes. These ambient techniques are commonly implemented on benchtop systems, but their potential application with miniature mass spectrometers for in situ measurements is even more powerful. These applications require that attention be paid to

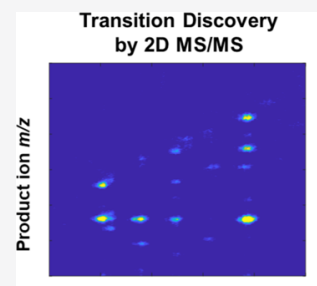

Precursor ion $\mathrm{m} / \mathrm{z}$ 个 MS/MS Duty Cycle
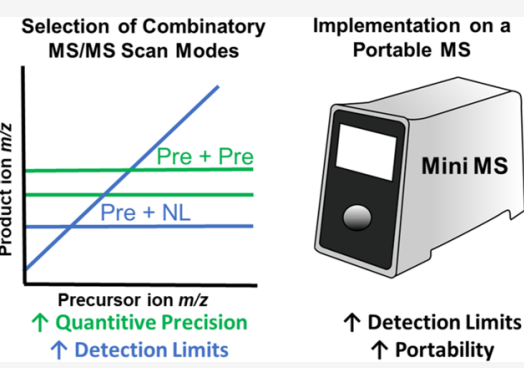
tailoring the mass spectrometric methodology for the on-site operation. In this study, combinations of scan modes are employed to efficiently determine what tandem mass spectrometry (MS/ MS) operations are most useful for detecting sulfonamides using a miniature ion trap after ionization. First, mixtures of representative sulfonamide antibiotics were interrogated using a 2D MS/MS scan on a benchtop ion trap in order to determine which class-specific fragments (ionic or neutral) are shared between the sulfonamides and thus have diagnostic value. Then, three less-used combination scans were recorded: (i) a simultaneous precursor ion scan was used to detect both analytes and an internal standard in a single ion injection event to optimize quantitative performance; (ii) a simultaneous precursor/neutral loss scan was used to improve detection limits; and finally, (iii) the simultaneous precursor/neutral loss scan was implemented in a miniature mass spectrometer and representative sulfonamides were detected at concentrations as low as $100 \mathrm{ng} / \mathrm{mL}$ by nano-electrospray and $0.5 \mathrm{ng}$ absolute by paper spray ionization, although improvements in the stability of the home-built instrumentation are needed to further optimize performance.

\section{INTRODUCTION}

The rapid emergence of antibiotic-resistant pathogens has developed into a global health crisis. ${ }^{1}$ A major contributor to the development of resistant strains is the misuse or overuse of antibiotics in the agriculture and food industry. Such overuse of antibiotics in the livestock industry has resulted in strict regulations requiring the monitoring of antibiotic use. For example, the European Union has set the maximum residue limit (MRL) for sulfonamides in all food products at $100 \mu \mathrm{g} /$ $\mathrm{kg} .{ }^{2}$ However, adequate food safety testing is a complex logistic operation, where samples are collected on-site and then brought to a central laboratory for analysis. Because the great majority of those samples, fortunately, test negative for contamination, time and resources could be saved by eliminating them at the sampling site-thus only collecting the suspect samples for further confirmatory analysis in the laboratory. The standard method of quantifying antibiotics in food products is extraction followed by liquid chromatography. ${ }^{3,4}$ However, this is a time-consuming process that is not well-suited to on-site measurements with portable instrumentation. In order to overcome such limitations, appropriate techniques for direct sampling of analytes with minimal sample cleanup ${ }^{5}$ have been developed, and these methods have been used on a variety of samples and have also been demonstrated on portable instrumentation. ${ }^{6}$ However, the majority of such efforts have focused on the front side, namely, ambient ionization, rather than investigating the possibilities of optimizing the subsequent mass analyzer (scan operations). Dedicated development in the scan operations is needed in order to further characterize the species generated from (ambient) ionization methods.

Ambient ionization comprises a family of methods that allow in situ analysis to be performed using mass spectrometers of a range of sizes. ${ }^{7}$ Much research has been done on various ionization techniques optimizing performance for specific analyses. ${ }^{8,9}$ The flexibility afforded by changing many physical or chemical parameters while performing ambient ionization has given practitioners many options to tailor the ion source to maximize performance. Examples of ionization methods for the antibiotic detection in complex matrixes include the following:

Received: July 3, 2021

Accepted: September 26, 2021

Published: October 7, 2021 
electrospray ionization (ESI) for milk samples, ${ }^{10-12}$ nanoelectrospray ionization (nESI) for infant formula, ${ }^{13}$ paper spray for biofluids, ${ }^{14}$ microwave plasma torch (MPT) for honey, ${ }^{15}$ and extractive electrospray ionization (EESI) for viscous cosmetic products, ${ }^{16}$ with sample preconcentration and cleanup methods, such as solid-phase microextraction (SPME), ${ }^{10,11,13}$ solid-phase extraction (SPE), ${ }^{12}$ or simplified extraction ${ }^{14}$ often being necessary to compensate for the lack of chromatographic separation. Requiring minimal sample preparation and time, ambient ionization is most impactful when coupled to mass spectrometers that can be operated outside an analytical laboratory.

Although ambient ionization is advantageous in performing analysis of the sample, it suffers from several limitations as well. The lack of sample pretreatment and separation before ionization results in a complex set of ions entering the MS all at once, reducing the selectivity of an analysis and necessitating tandem mass spectrometry (MS/MS) operations. Moreover, in the absence of sample cleanup, ion suppression, which is known to especially influence electrospray processes, can become a serious limitation. ${ }^{17}$ Although selective enrichment in ambient ionization methods can be employed to partially overcome such issues, selectivity remains a major challenge, which cannot be easily overcome by ambient ionization alone. Therefore, it is imperative that tandem mass spectrometry be performed on-site as well. Especially valuable are scan modes that allow for the analysis of a class of compounds, based on a shared product ion or common neutral loss when screening for larger families of molecules, such as the sulfonamides. Simply put, the mass spectrometer must be able to quickly determine what ionic species are of interest, based on specific fragmentation patterns. However, this poses another dilemma, which is that traditionally space-dependent tandem mass spectrometers, e.g. quadrupole mass filters, allow for the most flexibility in the types of MS/MS scans available, whereas time-dependent tandem mass spectrometers, e.g. quadrupole ion traps, are best suited for miniaturization due to mild vacuum requirements and requiring only a single mass analyzer.

Previously, we have demonstrated that data-independent MS/MS scans, first implemented using benchtop ion traps, can be used with miniature mass spectrometers to optimize sample use and analysis time. ${ }^{18,19}$ These MS/MS scans are most readily coupled to miniature mass spectrometers by utilizing a discontinuous atmospheric pressure interface (DAPI), which introduces both ions and neutrals over a small time interval in order to reduce vacuum pump requirements. ${ }^{20}$ Instruments utilizing the DAPI require significant pump-down time after sample introduction before mass analysis, thereby increasing the total scan-to-scan time. Remarkably, a single low-resolution quadrupole ion trap allows for implementation of precursor ion, neutral loss, and two-dimensional scans. The dataindependent one- and two-dimensional scans are more timeefficient than the traditional ion trap product ion scan when examining multiple precursor ions containing the same functional group, because product ion scans require ion isolation for each precursor ion interrogated, thereby requiring multiple ion injections. The precursor ion and neutral loss scans only require one ion injection per fragment (ionic or neutral) interrogated, as no isolation is required.

These novel ion trap scans are useful in the case of sulfonamide antibiotics, as there are two substituents that vary in this compound class. The sulfur-bonded substituent is typically a para-aniline functional group, while the nitrogen substituent varies depending on the specific biological activity, with the most common moieties being nitrogen-containing heteroaromatics. All 17 sulfonamide antibiotics studied here contain a para-aniline moiety. This conserved moiety is the basis for the untargeted detection of this class of compound using MS/MS scan modes, which is therefore very suited to demonstrate the value of optimizing the MS/MS transitions for on-site detection of classes of food contaminants. The conserved moiety and how the molecules containing this moiety fall into the two-dimensional MS/MS data space are shown in Figure 1. It should be noted that combinations of

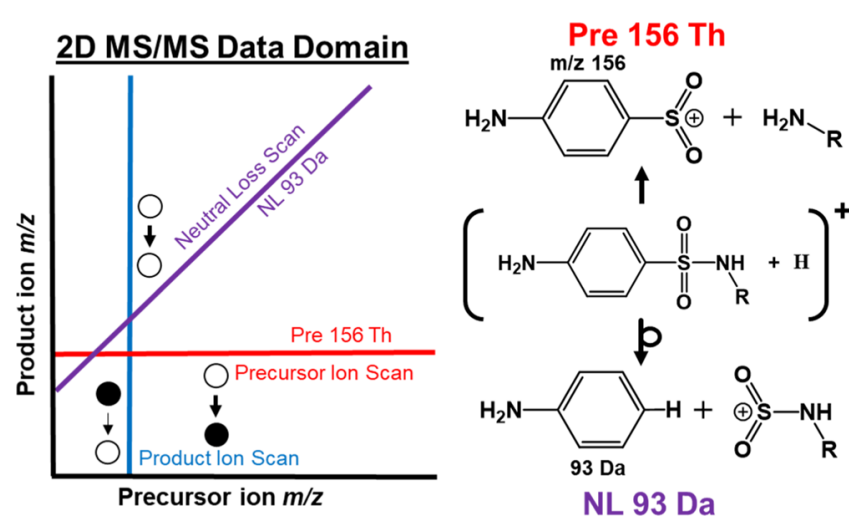

Figure 1. 2D MS/MS data domain with one-dimensional scan lines depicted. Also shown are the routes to two common fragments of $p$ aniline sulfonamides: neutral loss (NL) $93 \mathrm{Da}$ or formation of product ion $m / z 156$.

one-dimensional MS/MS scans are also useful, as demonstrated previously. ${ }^{21}$ Logical operations can be performed by combining scans, in particular, the AND/OR operation can be performed by simultaneously performing two scans. ${ }^{22}$ These simultaneous scans can be tailored for certain analytes, and in this study, we show how the combinations of scans, specifically (i) two precursor scans and (ii) a precursor and a neutral loss scan can be tailored to prioritize quantitative precision or detection limits, respectively. The latter combination scan, because of its lower detection limit using benchtop instrumentation, was then implemented on a miniature mass spectrometer to demonstrate the feasibility of detection of such antibiotics on-site at concentrations of practical significance.

\section{EXPERIMENTAL SECTION}

Chemicals. Sulfadiazine, sulfamerazine, sulfamethizole, sulfamethoxazole, sulfamethoxypyridazine, sulfapyridine, sulfaquinoxaline, sulfathiazole, dapsone, sulfisoxazole, sulfamonomethoxine, sulfadimethoxine, sulfamoxole, sulfacetamide, sulfaphenazole, trimethoprim, sulfadimidine, and sulfachlorpyridazine were provided by Riedel de Haen (Seelze, Germany). The isotope labeled sulfadimethoxine- $d_{6}$ was purchased from Sigma-Aldrich (St. Louis, US). The isotope labeled dapsone- $d_{8}$ was obtained from Toronto Research (Toronto, ON, Canada). Sulfapyridine $-{ }^{13} \mathrm{C} 6$, sulfamerazine $-{ }^{13} \mathrm{C} 6$, sulfamethizole- ${ }^{13} \mathrm{C} 6$, sulfamethoxypyridazine- $d_{3}$, sulfachlorpyridazine $-{ }^{13} \mathrm{C} 6$, sulfisoxazole- ${ }^{13} \mathrm{C} 6$, and sulfaquinoxaline $-{ }^{13} \mathrm{C} 6$ were purchased from Witega (Berlin, Germany). Each sulfonamide was diluted in methanol to create stock solutions at $1 \mathrm{mg} / \mathrm{mL}$. For the $2 \mathrm{D}$ MS/MS scan, three mixtures containing ten sulfonamides each, whose light-isotope structures and precursor $m / z$ values can be 
found in the Supporting Information (Figure S1, and Table S1, respectively), were diluted to $1 \mu \mathrm{g} / \mathrm{mL}$ in $1: 1 \mathrm{MeOH} / \mathrm{H}_{2} \mathrm{O}$ and $0.1 \%$ formic acid. For quantitation by simultaneous precursor ion scans, five sulfonamides, sulfapyridine, sulfamerazine, sulfadimidine, sulfaquinoxaline, and sulfadimethoxine, were combined and diluted to $1000,800,600,400,200,100$, and 50 $\mathrm{ng} / \mathrm{mL}$ in a $1: 1 \mathrm{MeOH} / \mathrm{H}_{2} \mathrm{O}$ and $0.1 \%$ formic acid solution containing the corresponding isotopically labeled internal standards at $400 \mathrm{ng} / \mathrm{mL}$. A significant degradation of the analytes, identified by a strong decrease in ion signal, was observed over the course of a day, so further experiments were performed using acetonitrile as a solvent (i.e., the five sulfonamides were diluted to working concentrations by diluting stock solutions of the mixtures in acetonitrile). Fresh solutions were prepared daily.

Instrumentation. The modified Finnigan LTQ mass spectrometer (San Jose, CA) and modified Mini 12 mass spectrometer capable of performing single-analyzer MS/MS scans have been described previously. ${ }^{18,23}$ The precursor ion and neutral loss scan mode have been previously implemented in one of two possible configurations in a quadrupole ion trap, where the radio frequency (RF) trapping voltage is either constant $^{24,25}$ or varied. ${ }^{26,27}$ For the scan modes demonstrated here, the RF trapping voltage must be kept constant so that ions maintain a constant secular frequency throughout the scan. This is accomplished in the benchtop LTQ instrument by substituting the instrument's RF voltage control with an external waveform generator. This implementation does not have any feedback and results in a slight drift in the output RF voltage. The voltage drift results in an ion having slightly different secular frequencies throughout the experiment. Frequent calibration and re-optimization, therefore, are required for optimal performance. The miniature mass spectrometer has active RF voltage feedback, resulting in far less drift, so no re-optimization was required. Both instruments have been previously modified so that external waveform generators can be inductively coupled to the $\mathrm{x}$ - and $\mathrm{y}$-rod pairs of the ion trap. The bath gas in the modified LTQ was nitrogen, while the miniature instrument used the air introduced during ion introduction when the DAPI valve was open.

Nano-electrospray ionization was accomplished by pipetting a liquid sample into a borosilicate glass capillary ( $1.5 \mathrm{~mm}$ o.d., $0.86 \mathrm{~mm}$ i.d.) from Sutter Instrument Co. pulled to a $5 \mu \mathrm{m}$ tip diameter by a Flaming/Brown micropipette puller (model P97, Sutter Instrument Co.). The loaded tip was then placed in a nano-spray electrode holder (model ESW-M15P Warner instruments, Hamden, CT). A $1.5 \mathrm{kV}$ potential was applied to the electrode holder with the tip of capillary placed $\sim 1 \mathrm{~cm}$ away from the mass spectrometer inlet to generate nanoelectrospray. Paper spray mass spectrometry was accomplished by applying $5 \mu \mathrm{L}$ of diluted solutions in the center of a Whatman 1 (Fisher Scientific) paper substrate with an isosceles triangle geometry with a width of 1.5 and $2 \mathrm{~cm}$ long. The paper substrate was held $5 \mathrm{~mm}$ away from the mass spectrometer inlet by a stainless-steel alligator clip (CAL$\mathrm{HAWK}$ ) and was allowed to dry. Electrospray was generated by the application of $45 \mu \mathrm{L}$ of $9: 1 \mathrm{ACN} / \mathrm{H}_{2} \mathrm{O}$ to the back of the paper substrate and a potential of $3.5 \mathrm{kV}$ to the attached alligator clip. Copper alligator clips were avoided as the presence of copper/acetonitrile adducts was observed in the mass spectrum.
Scan Modes. In order to perform single-analyzer MS/MS scans, the ion trap was operated at a constant RF voltage to allow ions to maintain a constant secular frequency. ${ }^{28}$ The ions can be resonantly excited in either $\mathrm{x}$ - or $\mathrm{y}$-dimension by applying an auxiliary waveform containing their particular secular frequencies. The resonant ions can be ejected or fragmented by increasing or decreasing the applied amplitude of the applied ac waveform, respectively. A fragmentation waveform that results in a linear mass/time calibration was created in a previously described MATLAB program. ${ }^{28}$ This waveform is applied across the y-electrode pair (Figure 2), and
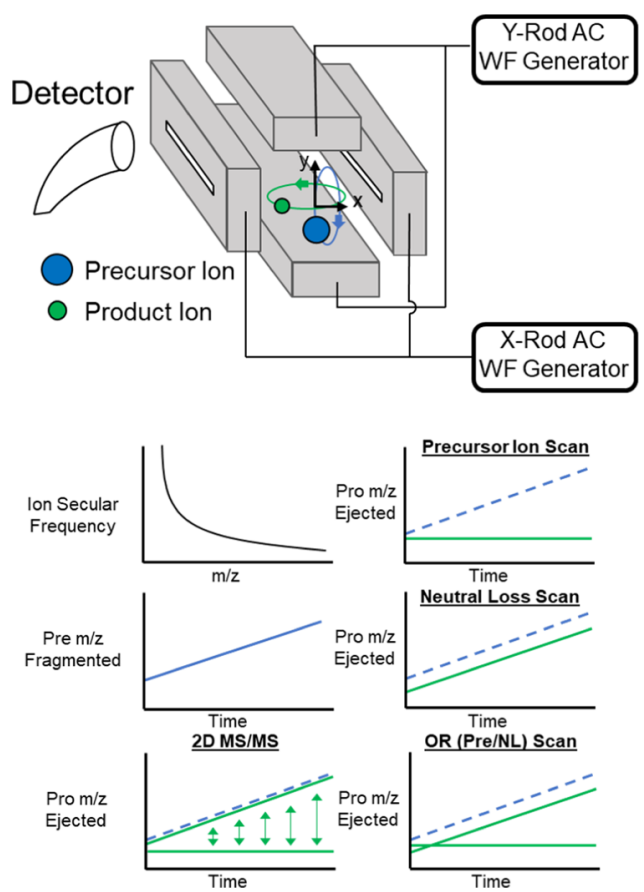

Figure 2. Orthogonal double resonance performed using a rectilinear ion trap. The y-rod waveform generator is used to successively fragment precursor ions of increasing the $\mathrm{m} / z$ value, while the x-rod continuously ejects either a constant $\mathrm{m} / z$ (precursor ion scan) or constant mass offset (neutral loss scan). To record the 2D MS/MS spectrum, the waveform applied to the $\mathrm{x}$-rod pair was continuously swept over the possible product ion mass range, while that applied to the y-rods was incremented. The OR scan is performed by combining individual precursor ion/neutral loss waveforms.

it fragments precursor ions of increasing $\mathrm{m} / \mathrm{z}$ over the entire scan. The resulting product ions can then be rapidly ejected for detection by applying another waveform to the orthogonal rod pair (Figure 2). The combination of precursor and product ion fragmentation/ejection waveforms determines which of the MS/MS scans is being performed. A precursor ion scan uses a fixed ejection frequency, resulting in a single product ion being continuously ejected throughout the scan. ${ }^{25}$ Ions are detected when a precursor ion is fragmented to produce an ion whose secular frequency matches the applied ejection frequency. Neutral loss scans are performed by applying the same waveform for ejection as that used for fragmentation but with a fixed time offset so that a fixed mass offset is established. ${ }^{29}$ An additional waveform is applied in the time between the fragmentation and ejection waveform so that unfragmented precursors are not detected. The $2 \mathrm{D}$ MS/MS scan is performed by rapidly sweeping through all possible product ion frequencies and iterating this sweep continuously to 

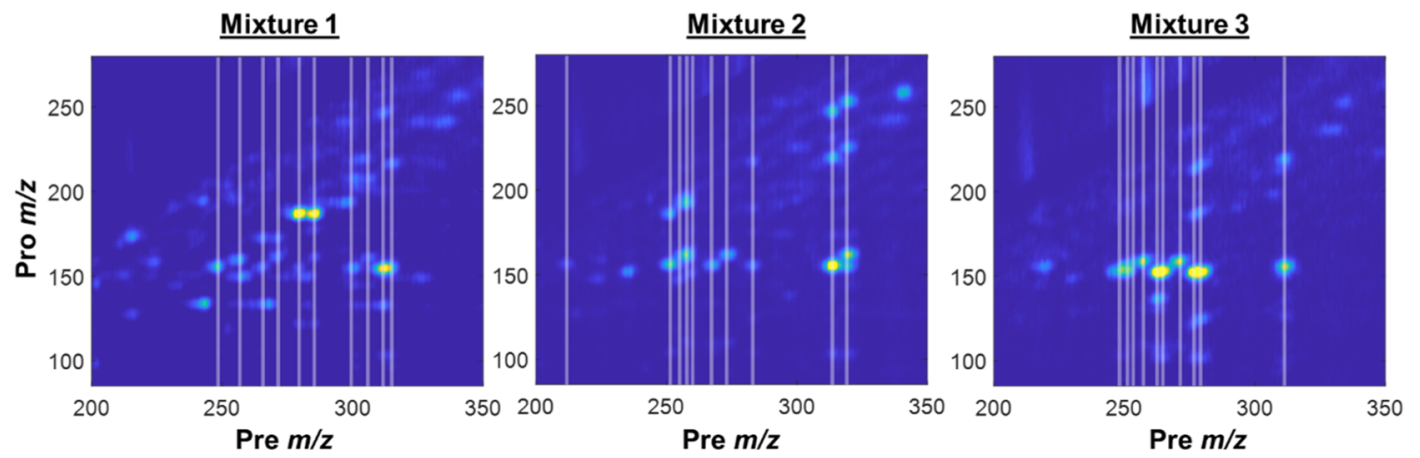

Figure 3. 2D MS/MS scan recorded using the benchtop instrument for three 10-component sulfonamide mixtures, each at $1 \mu \mathrm{g} / \mathrm{mL}$. The spots are aligned to form horizontal and diagonal lines which correspond to precursor ion scan lines [constant product (Pro) values] and neutral loss scan lines, respectively. Vertical lines added to the figure indicate predicted $m / z$ values for $[\mathrm{M}+\mathrm{H}]^{+}$product ion scans [constant precursor (Pre) values] of each of the ten components. The composition of each mixture can be found in the Supporting Information, Table S1.

preserve precursor/product ion relationships. ${ }^{23}$ The observed output is spliced into individual product ion sweeps, and those individual sweeps are combined into a single matrix in MATLAB. The simultaneous combinations of scans were accomplished by applying product ion ejection waveforms at the same time as those corresponding to the individual MS/ MS scans. ${ }^{21}$

\section{RESULTS AND DISCUSSION}

Discovery of Diagnostic Transitions by 2D MS/MS. Data-independent MS/MS scan modes require a characteristic fragment that can be used to identify a particular functional group. If the characteristic fragment retains a charge, it can be observed with a scan of a single product ion. If instead the charge resides within the nonspecific fragment, the characteristic neutral fragment can be observed in the neutral loss scan (constant mass difference between the detected product ion and the fragmented precursor ion). The location of the charge site for an ionized analyte with many functional groups is dependent on thermochemical properties of the various charge sites. $^{30,31}$ The charge site location can influence the fragmentation pattern, determining if the characteristic fragment is charged or neutral. ${ }^{32}$ Rearrangements during fragmentation can further complicate assessing where the charge is retained. ${ }^{33}$

In order to determine the nature and distribution of characteristic fragment ions for various antibiotics, three mixtures of sulfonamides and their corresponding isotope labeled standards were interrogated using the 2D MS/MS scan in which all precursor ions and their subsequent product ions are detected. The three $2 \mathrm{D}$ MS/MS spectra obtained are shown in Figure 3. The characteristic fragments for the sulfonamides are seen in the 2D MS/MS spectrum, which are aligned horizontally and diagonally, representing conserved, charged, and neutral fragments, respectively. The fragment that was obtained for all sulfonamides and thus has the highest diagnostic value was due to the cleavage of the sulfur-nitrogen bond to form product ion $m / z 156$ (Figure 1) for light-isotope sulfonamides and heavier product ions for heavy-isotope standards depending on the location of the heavy isotopes. The second most commonly occurring fragment was due to the neutral loss of $93 \mathrm{Da}$, which corresponds to dissociation at the sulfur-carbon bond. This fragmentation pathway involves a rearrangement requiring a proton transfer to form neutral aniline $(93 \mathrm{Da})$. The appearance of this neutral loss competes with other previously observed rearrangements such as the neutral loss of $66, \mathrm{H}_{2} \mathrm{SO}_{2}$, and the formation of product ion $\mathrm{m} /$ $z 108$, which requires breaking a sulfur-oxygen bond and the formation of a carbon-oxygen bond. ${ }^{34}$ Interestingly, for three pyrimidine sulfonamides, each addition of a methyl group increases the likelihood of fragmenting to form neutral aniline over p-aniline sulfonyl cation, viz., the fragment resulting from neutral loss of $93 \mathrm{Da}$ versus the product ion $\mathrm{m} / z 156$ (Figure S2).

Two-Dimensional Scan Performance. The 2D MS/MS scan provides a comprehensive view of the ionic landscape, but the data suffers from poorer mass resolution compared to traditional ion trap product ion scans. The composition of the mixtures shown in Figure 3 was chosen to decrease spectral overlap of sulfonamide precursor ions. In real samples, higher resolution may be required in order to improve specificity. In order to improve mass selectivity, the mass scan rate can be decreased by reducing the mass range, while keeping the scan time constant or by acquiring the same mass range over a longer time period. The extreme case of this is by reducing the mass range, so that a single $\mathrm{m} / z$ is being monitored, in other words, a reduction in mass dimensionality.

In order to assess the tradeoff between mass dimensionality and mass resolution, a comparison between an extracted precursor ion scan and the equivalent single-analyzer precursor ion scan is shown in Figure 4b. The peaks obtained in the onedimensional scan are better resolved than the equivalent peaks extracted from the 2D MS/MS scan. Specifically, the full width at half-maximum (fwhm) of the precursor ion $\mathrm{m} / z 250$ is 4.5 in the $2 \mathrm{D} \mathrm{MS} / \mathrm{MS}$ scan and 1.6 in the precursor ion scan. The difference in mass resolution is due to the fact that the 2D MS/ MS scan must be performed on approximately 250 possible product ion $\mathrm{m} / z$ values in $1.5 \mathrm{~ms}$, whereas the precursor ion scan devotes that same time to a single $\mathrm{m} / z$ value. In certain analyses, the greater mass resolution of the precursor ion scan would be more useful even at the cost of the loss of additional MS/MS information. The sulfonamide analytes being monitored are expected to be found in many different food products, which contain very different chemical interferents. The difference in chemical complexity dictates that a mass spectrometer be able to operate in multiple configurations.

Simultaneous One-Dimensional Scan Modes. The combination of multiple MS/MS scans provides flexibility in detecting multiple product ions belonging to the same chemical class. The detection of all the sulfonamides is accomplished using the characteristic $\mathrm{m} / z 156$ product ion. However, the carbon-13 labeled standards used for quantifi- 


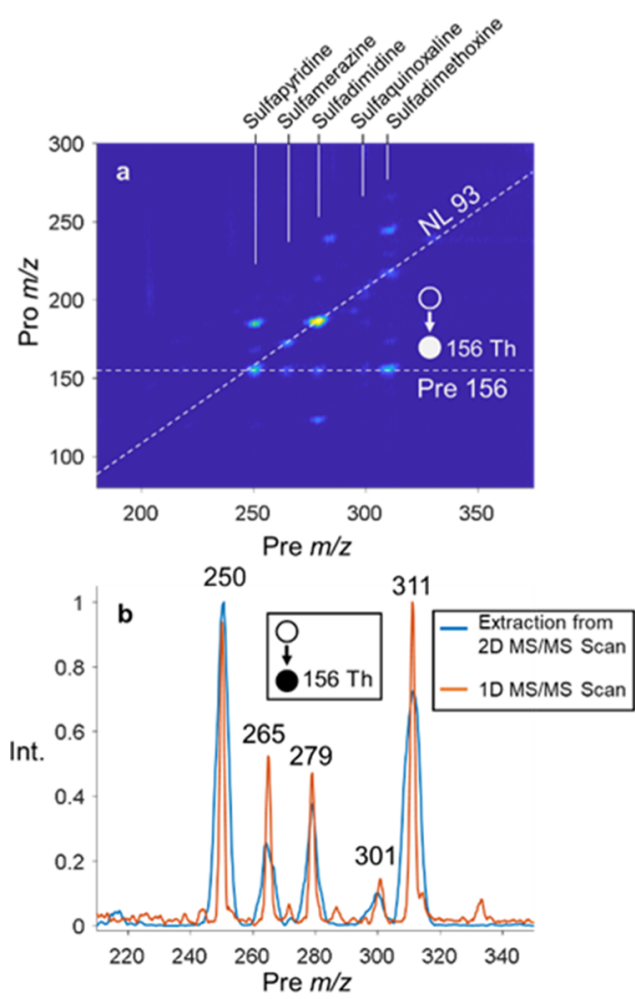

Figure 4. (a) 2D MS/MS spectrum of five sulfonamides with Pre and NL scan lines overlaid. (b) Comparison of the extracted precursor ion scan (blue) and 1D precursor ion scan (orange) for product ion $\mathrm{m} / \mathrm{z}$ 156. Both $(\mathrm{a}, \mathrm{b})$ were acquired from a benchtop ion trap mass spectrometer. cation of the sulfonamides do not fragment to the same product ion $m / z$ as the light-isotopologues. Instead, these labeled standards fragment to the product ion $\mathrm{m} / z 162$. Traditional analysis would require an additional scan to record the internal standard, thereby doubling the analysis time. Furthermore, by detecting the standard and analyte in separate ion injections, fluctuations in the ion current can decrease quantitative precision, which are common in ambient ionization. By combining two precursor ion scans for the common product ions of both the analyte and internal standard, better quantitative performance would be expected.

The quantification of five sulfonamides by the simultaneous precursor scan for $m / z 156$ and 162 is shown in Figure 5. The analyte signal is normalized using the intensity of the internal standard to correct for deviations in the spray current. After normalization to the internal standard intensity, the $R$-squared coefficients of the linear regression models improved on average $12 \%$ for the five analytes (Table S2). However, large sample-to-sample variation was observed across the three calibration sets evaluated. This was reflected in an adequate linear response within each calibration set, but with significant variation in the signal intensity for both analytes and internal standards across different sets. The variation was attributed to a drift over time in the amplitude of the voltage used to trap ions. This drift results in ions having slightly different secular frequencies and not being completely in resonance with the frequency used to eject product ions, thereby decreasing ejection efficiency and lowering sensitivity. One solution to this problem is to further improve the modified instrument's electronics to minimize the error in the output voltage. A more immediately accessible approach is to normalize each measurement to the average response of the internal standard across
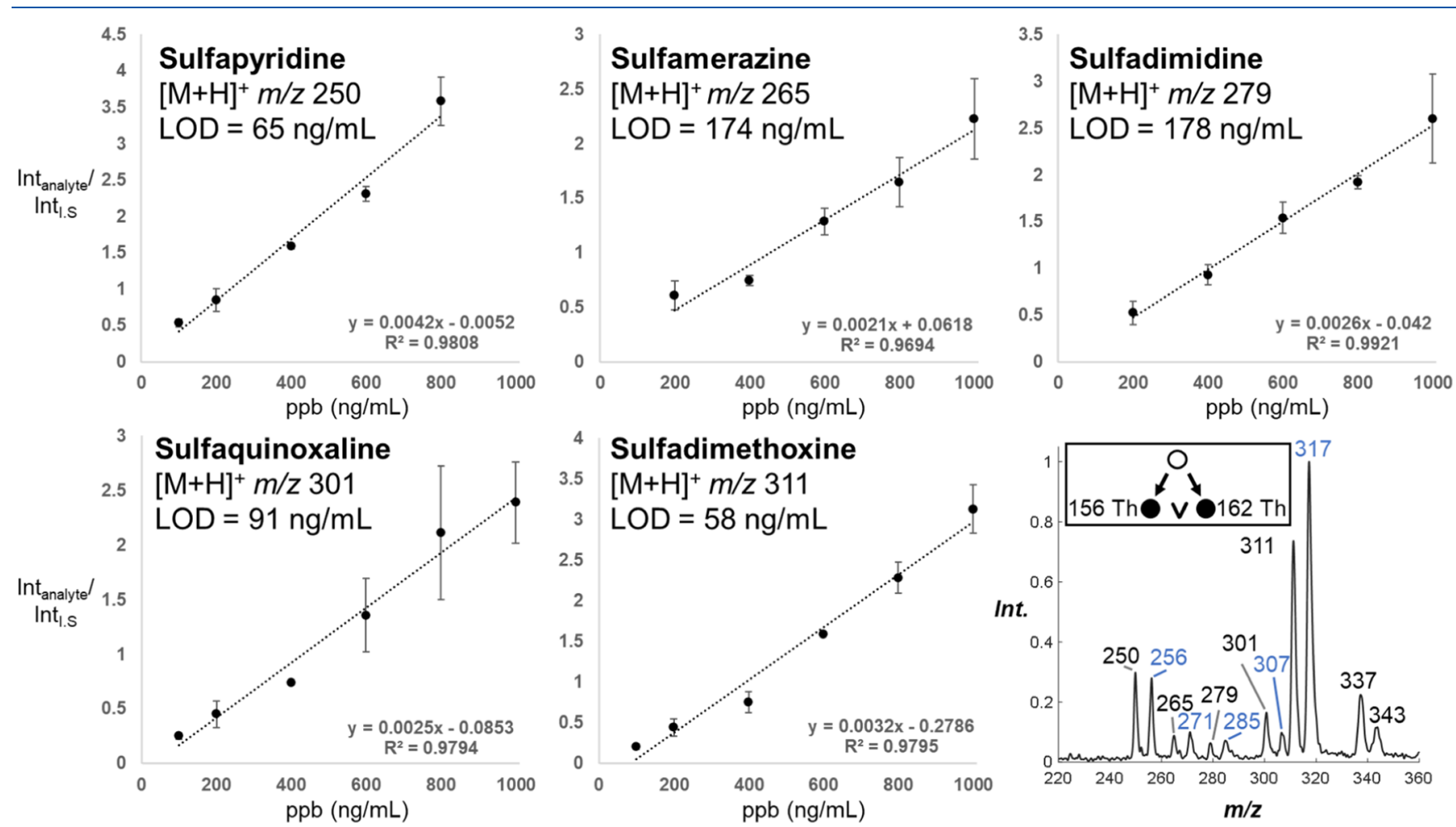

Figure 5. Quantification of five sulfonamides using internal standard normalization from simultaneous precursor ion scans acquired from a benchtop mass spectrometer. Bottom right: example of simultaneous OR precursor ion scan for $m / z 156$ (analyte, black) and 162 (IS, blue) at 200 $\mathrm{ng} / \mathrm{mL}$. 
each of the three replicates. As expected, this second normalization has no significant effect on the linearity of the response, but it does decrease the relative standard deviation calculated across replicates by $15 \%$ (in average for the five analytes and all the calibration standards), compared to nonnormalized data (Table S2). It should be noted that by detecting an additional product ion, it is possible to detect interferents that would otherwise not be detected, thus decreasing specificity. This operation is considered a simultaneous OR scan because the intensity observed for each $\mathrm{m} / z$ can come from one product ion or the other product ion. If the intensities attributed from either product ion can be obtained, it is possible to include more sophisticated logical operations such as the AND, XOR (exclusive OR), and BUT NOT scans.

Although the combination of precursor ion scans improves quantitative precision, many on-site chemical detection experiments prioritize sensitivity and selectivity over quantitative accuracy as suspect samples are sent to laboratories for confirmatory analysis. It can be seen that two of the sulfonamides, sulfamerazine $(m / z 265)$ and sulfadimidine $(\mathrm{m} / z 279)$, have notably high limit of detections, 174 and 178 $\mathrm{ng} / \mathrm{mL}$, respectively, compared to the other analytes. To improve sensitivity, the supplemental waveform used to eject the product ions of $\mathrm{m} / \mathrm{z} 162$ (the product ion used for detecting the internal standard) is exchanged for one that ejects the product ions that result from a neutral loss scan of 93 $\mathrm{Da}$ from the fragmenting precursor ion. Note that the integration of two product ions into one detection channel (i.e., the combination precursor/neutral loss scan) results in greater signal than each of the individual scans, as demonstrated in Figure 6. It is expected, however, that as

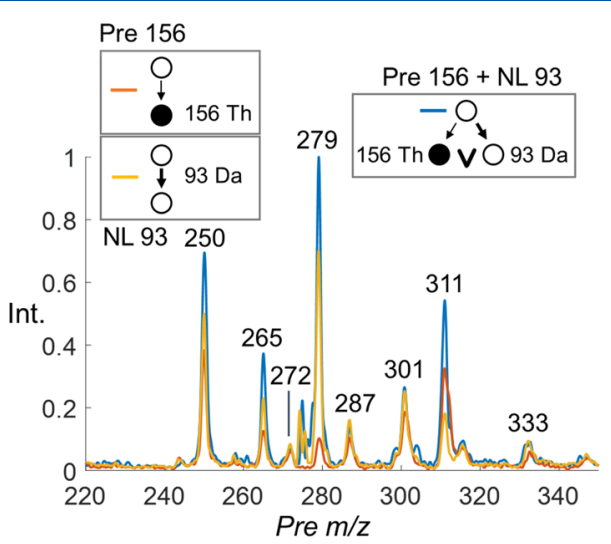

Figure 6. Comparison of individual precursor ion (orange), neutral loss (yellow) scan, and simultaneous OR precursor/neutral loss scan (blue) of a solution containing five sulfonamides at $200 \mathrm{ng} / \mathrm{mL}$ acquired from a benchtop mass spectrometer. The peaks at $m / z 272$, 287, and 333 correspond to sodiated sulfonamide species.

the overall signal is increased by adding two channels together, the overall noise should increase as well. Nonetheless, this increase in noise is minor compared to the increase in signal and an improvement in signal/noise ratio is observed for all the analytes at $100 \mathrm{ng} / \mathrm{mL}$ (Figure S3). As all signal/noise ratios are greater than three, it is assumed that these analytes would be detected at concentrations below the MRL in matrices when combined with an appropriate ion source. It is noteworthy that all three spectra show the same peaks, consistent with the fact that the simultaneous scan used here is an OR function, but also indicating that these precursor ion (for product ion $\mathrm{m} / \mathrm{z} 156$ ) and neutral loss (of $93 \mathrm{Da}$ ) scans are highly diagnostic for all the sulfonamides in this set.

Detection of Sulfonamides Using a Miniature Mass Spectrometer. The benchtop performance of the combination scans has allowed flexibility in prioritizing either quantitative performance or improved detection limits. The utilization of these scan modes for the on-site detection of sulfonamides should be tailored specifically to the analytical demands. For example, the ambient detection of these antibiotics would require direct analysis from a sample, such as milk, without separation. Despite the typical decrease in quantitative performance of these ambient ionization techniques when compared to traditional analytical workflows (i.e., extraction, separation and then ionization), there are many situations in which rapid on-site analysis using only relative intensities between multiple analytes is preferred if a choice must be made for high quality quantitation. ${ }^{35,36}$

For the on-site detection of antibiotics in food products, it is likely that improved detection limits would be more important than quantitative precision. For this reason, the simultaneous precursor/neutral loss scan was implemented on a homebuilt miniature mass spectrometer to prioritize detection limits. A comparison of the performance of the homebuilt mass spectrometer to the benchtop mass spectrometer can be found in Figure 7. For four of the five analytes, an

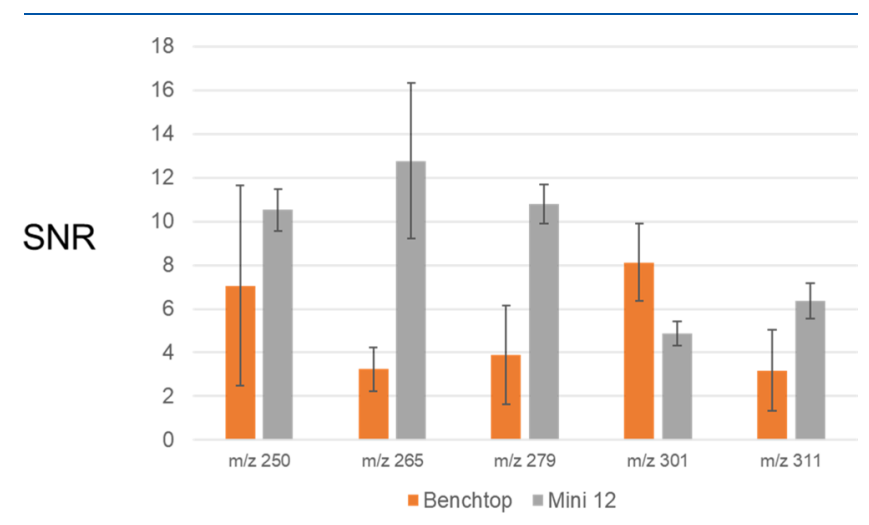

Figure 7. Comparison of the signal/noise ratio (SNR) for the simultaneous precursor/neutral loss scan on the benchtop mass spectrometer (orange) and miniature mass spectrometer (gray) for the mixture of five sulfonamides. Note that $\mathrm{m} / \mathrm{z} 301$ contains contributions from both protonated and sodiated species.

improvement of the signal/noise ratio was observed across three replicates at the $100 \mathrm{ng} / \mathrm{mL}$ level. This is expected as previous comparisons between the two instruments using similar scans showed greater sensitivity on the miniature instrument, ${ }^{18,19}$ a phenomenon mostly attributed to the miniature instrument operating at higher pressure, thereby increasing fragmentation efficiency due to greater momentum transfer into the ion.

Nano-electrospray was used in previous experiments as it was able to generate stable ion currents for longer periods than paper spray ionization. This was done to better characterize the performance of the MS/MS scan modes. To demonstrate the feasibility of detecting antibiotic sulfonamides in a miniature instrument, the five analytes were detected at various amounts by paper spray ionization. This was accomplished by pipetting $5 \mu \mathrm{L}$ of solutions containing the analytes at 1000,500 , and 100 $\mathrm{ng} / \mathrm{mL}$ on a triangular paper substrate. In one successful 
experiment, the five analytes were detected at each concentration (Supporting Information, Figure S4). However, the results presented in Figure S4 represent the only successful attempt, and even at the highest concentration, multiple replicates were unsuccessful. Similar difficulties in consistency were observed when using a commercial benchtop instrument. To a lesser extent, difficulties with spray consistency were also observed in nano-electrospray. Earlier experiments demonstrated that the ionization of the protonated analytes is drastically affected by nano-electrospray capillary geometry (Supporting Information, Figure S5). The difficulty of ionizing these analytes emphasizes the need for continued development in ambient ionization ion sources.

\section{CONCLUSIONS}

This work establishes a framework for interrogating (new) classes of molecules by data-independent MS/MS with respect to analytical metrics. First, representative molecules are chosen and detected by a 2D MS/MS scan which identifies all precursor/product ion relationships. From the 2D MS/MS spectrum, the most informative 1D MS/MS scan lines can be identified. By spending the entire scan time on the identified 1D MS/MS scan line (Pre 156), greater mass resolution is obtained (fwhm 4.5 for the 2D MS/MS scan and 1.6 for the precursor ion scan). Furthermore, individual 1D MS/MS scan lines can be combined simultaneously in order to prioritize either detection limits or quantitative precision. These 1D MS/ MS combinatory scans are most important when combined with ambient ionization and miniature mass spectrometers to make measurements on-site with minimal sample preparation. The simultaneous OR precursor/neutral loss scan was shown to have the best detection limit $(<100 \mathrm{ng} / \mathrm{mL})$ and was used to detect representative sulfonamides on a miniature mass spectrometer. Further improvements on the reproducibility and direct interfacing capability of ambient ionization sources, such as paper spray, will complement the approach reported here to allow robust and reliable on-site analysis with miniature mass spectrometry.

\section{ASSOCIATED CONTENT}

\section{(s) Supporting Information}

The Supporting Information is available free of charge at https://pubs.acs.org/doi/10.1021/acs.analchem.1c02790.

Sulfonamide structures; neutral loss/product ion ratio for pyrimidine sulfonamides; comparison of SNR for precursor ion, neutral loss, and combination scans; paper spray of sulfonamides on miniature MS with combined precursor ion/neutral loss scan; nESI of sulfonamides from capillaries with and without filament; overview of sulfonamide mixtures; and effects of signal normalization for calibration curves on benchtop instrument (PDF)

\section{AUTHOR INFORMATION}

\section{Corresponding Authors}

R. Graham Cooks - Department of Chemistry, Purdue University, West Lafayette, Indiana 47907, United States; ○ orcid.org/0000-0002-9581-9603; Email: cooks@ purdue.edu

Gert IJ. Salentijn - Laboratory of Organic Chemistry, Wageningen University, Wageningen 6708 WE, The Netherlands; Wageningen Food Safety Research (WFSR), Wageningen University \& Research, Wageningen 6700 AE,
The Netherlands; 이이.org/0000-0002-2870-9084; Email: gert.salentijn@wur.nl

\section{Authors}

Lucas J. Szalwinski - Department of Chemistry, Purdue University, West Lafayette, Indiana 47907, United States; (1) orcid.org/0000-0002-1145-0694

Yanyang Hu - Department of Chemistry, Purdue University, West Lafayette, Indiana 47907, United States

Nicolás M. Morato - Department of Chemistry, Purdue University, West Lafayette, Indiana 47907, United States; ○ orcid.org/0000-0003-0218-407X

Complete contact information is available at:

https://pubs.acs.org/10.1021/acs.analchem.1c02790

\section{Notes}

The authors declare no competing financial interest.

\section{ACKNOWLEDGMENTS}

The authors acknowledge funding from Teledyne FLIR and NSF \#1906087. Greg Eakins, Mark Carlsen, and Ryan Hilger of the Jonathan Amy Facility for Chemical Instrumentation (JAFCI) are acknowledged for important modifications to the mass spectrometers. N.M.M. acknowledges support from the Charles H. Viol Memorial Fellowship. G.IJ.S. acknowledges financial support from the Dutch Ministry of Agriculture, Nature and Food Quality (project KB-37-002-005) and from the Dutch Research Council (NWO) (NWO talent programme, Veni grant 17328).

\section{REFERENCES}

(1) Yadav, S.; Kapley, A. Biotechnol. Rep. 2021, 29, No. e00604.

(2) Commission Regulation (EU) No 37/2010 of 22 December 2009 on pharmacologically active substances and their classification regarding maximum residue limits in foodstuffs of animal origin (2010) Official Journal L 015.

(3) Wu, J.; Li, Y.; Li, W.; Gong, Z.; Huang, X. Anal. Chim. Acta 2020, 1118, 9-17.

(4) Choi, S. Y.; Kang, H.-S. Food Anal. Methods 2021, 14, 12561268.

(5) Lawton, Z. E.; Traub, A.; Fatigante, W. L.; Mancias, J.; O’Leary, A. E.; Hall, S. E.; Wieland, J. R.; Oberacher, H.; Gizzi, M. C.; Mulligan, C. C. J. Am. Soc. Mass Spectrom. 2017, 28, 1048-1059.

(6) Jager, J.; Gerssen, A.; Pawliszyn, J.; Sterk, S. S.; Nielen, M. W. F.; Blokland, M. H. J. Am. Soc. Mass Spectrom. 2020, 31, 2243-2249.

(7) Huang, M.-Z.; Yuan, C.-H.; Cheng, S.-C.; Cho, Y.-T.; Shiea, J. Annu. Rev. Anal. Chem. 2010, 3, 43-65.

(8) Marić, M.; Marano, J.; Cody, R. B.; Bridge, C. Anal. Chem. 2018, 90, 6877-6884.

(9) Tillner, J.; Wu, V.; Jones, E. A.; Pringle, S. D.; Karancsi, T.; Dannhorn, A.; Veselkov, K.; McKenzie, J. S.; Takats, Z. J. Am. Soc. Mass Spectrom. 2017, 28, 2090-2098.

(10) Tian, H.; Liu, T.; Mu, G.; Chen, F.; He, M.; You, S.; Yang, M.; Li, Y.; Zhang, F. Talanta 2020, 219, 121282.

(11) Liu, Y.; Yang, Q.; Chen, X.; Song, Y.; Wu, Q.; Yang, Y.; He, L. Talanta 2019, 204, 238-247.

(12) Zhang, H.; Kou, W.; Bibi, A.; Jia, Q.; Su, R.; Chen, H.; Huang, K. Sci. Rep. 2017, 7, 14714.

(13) Guo, X.; Bai, H.; Ma, X.; Li, J.; Ren, Y.; Ouyang, Z.; Ma, Q. Anal. Chim. Acta 2020, 1098, 66-74.

(14) Suraritdechachai, S.; Charoenpakdee, C.; Young, I.; Maher, S.; Vilaivan, T.; Praneenararat, T. J. Agric. Food Chem. 2019, 67, 30553061.

(15) Jiang, T.; Peng, Z.; Xie, M.; Fang, X.; Hong, Y.; Huang, Z.; Gao, W.; Zhou, Z.; Li, L.; Zhu, Z. Anal. Methods 2020, 12, 535-543. 
(16) Liu, Y.; Zhang, X.; Ouyang, Y.; Hu, Z.; Ma, L.; Zhang, J.; Lin, J.;

Chen, H. J. Mass Spectrom. 2011, 46, 794-803.

(17) Annesley, T. M. Clin. Chem. 2003, 49, 1041-1044.

(18) Snyder, D. T.; Szalwinski, L. J.; Hilger, R.; Cooks, R. G. J. Am. Soc. Mass Spectrom. 2018, 29, 1355-1364.

(19) Szalwinski, L. J.; Cooks, R. G. Talanta Open 2021, 3, 100028.

(20) Gao, L.; Cooks, R. G.; Ouyang, Z. Anal. Chem. 2008, 80, 4026-4032.

(21) Snyder, D. T.; Szalwinski, L. J.; Cooks, R. G. Anal. Chem. 2017, 89, 11053-11060.

(22) Snyder, D. T.; Szalwinski, L. J.; Wells, J. M.; Cooks, R. G. Analyst 2018, 143, 5438-5452.

(23) Szalwinski, L. J.; Holden, D. T.; Morato, N. M.; Cooks, R. G. Anal. Chem. 2020, 92, 10016-10023.

(24) Johnson, J. V.; Pedder, R. E.; Yost, R. A. Int. J. Mass Spectrom. Ion Process. 1991, 106, 197-212.

(25) Snyder, D. T.; Cooks, R. G. J. Am. Soc. Mass Spectrom. 2017, 28, 1929-1938.

(26) McClellan, J. E.; Quarmby, S. T.; Yost, R. A. Anal. Chem. 2002, 74, 5799-5806.

(27) Snyder, D. T.; Szalwinski, L. J.; Schrader, R. L.; Pirro, V.; Hilger, R.; Cooks, R. G. J. Am. Soc. Mass Spectrom. 2018, 29, 13451354.

(28) Snyder, D. T.; Pulliam, C. J.; Cooks, R. G. Rapid Commun. Mass Spectrom. 2016, 30, 1190-1196.

(29) Snyder, D. T.; Cooks, R. G. Anal. Chem. 2017, 89, 8148-8155.

(30) Tian, Z.; Wang, X.-B.; Wang, L.-S.; Kass, S. R. J. Am. Chem. Soc. 2009, 131, 1174-1181.

(31) Warnke, S.; Seo, J.; Boschmans, J.; Sobott, F.; Scrivens, J. H.; Bleiholder, C.; Bowers, M. T.; Gewinner, S.; Schöllkopf, W.; Pagel, K.; von Helden, G. J. Am. Chem. Soc. 2015, 137, 4236-4242.

(32) Lermyte, F.; Theisen, A.; O'Connor, P. B. J. Am. Soc. Mass Spectrom. 2021, 32, 364-372.

(33) Demarque, D. P.; Crotti, A. E. M.; Vessecchi, R.; Lopes, J. L. C.; Lopes, N. P. Nat. Prod. Rep. 2016, 33, 432-455.

(34) Klagkou, K.; Pullen, F.; Harrison, M.; Organ, A.; Firth, A.; Langley, G. J. Rapid Commun. Mass Spectrom. 2003, 17, 2373-2379.

(35) Jarmusch, A. K.; Pirro, V.; Baird, Z.; Hattab, E. M.; CohenGadol, A. A.; Cooks, R. G. Proc. Natl. Acad. Sci. 2016, 113, 1486.

(36) Brown, H. M.; Pu, F.; Dey, M.; Miller, J.; Shah, M. V.; Shapiro, S. A.; Ouyang, Z.; Cohen-Gadol, A. A.; Cooks, R. G. Anal. Bioanal. Chem. 2019, 411, 7929-7933. 